\title{
Effect of Shield Insertion on Reducing Crosstalk Noise between Coupled Interconnects
}

\author{
Junmou Zhang and Eby G. Friedman \\ Department of Electrical and Computer Engineering \\ University of Rochester \\ Rochester, New York 14627-0231
}

\begin{abstract}
Placing shields around a victim signal line is a common way to enhance signal integrity while minimizing delay uncertainty. For two coupled interconnects with a shield between the lines, the coupling noise can produce a peak noise of $15 \%$ of $V_{d d}$ in a $0.18 \mu \mathrm{m}$ CMOS technology. A pseudo- $2 \pi R C$ model is used to develop an analytic estimate of the peak noise for shielded interconnects. The peak noise model is accurate within an average error of $4.4 \%$ as compared to SPICE. The effects of the shield width, length, separation between the shield and the signal, and the number of connections tieing the shield to ground on the overall crosstalk noise are described in this paper. Based on the peak noise model, a minimum number of ground connections for a target shield line with noise constraints is obtained. Inserting a shield line between two coupled interconnects is shown to be more effective in reducing crosstalk noise than increasing the physical separation.
\end{abstract}

\section{INTRODUCTION}

A $\mathrm{S}$ feature sizes are decreased to deep submicrometer dimensions, coupling capacitances can significantly affect circuit performance due to decreased interconnect spacing and increased interconnect thickness. Coupling noise has two deleterious effects on integrated circuits. When affecting a static signal, the noise can transiently destroy the logical information stored on the static node. This effect can ultimately result in an incorrect machine state stored within a latch, resulting in a functional failure. When noise occurs simultaneously with a switching event, the effect of noise is manifested as a change in the timing of the signal transition [1]. Several design and analysis techniques [2], [3] have been developed to manage delay uncertainty while minimizing signal noise.

Shielding in high speed digital circuits is an effective and common way to reduce crosstalk noise and signal delay uncertainty. In a $600 \mathrm{MHz}$ Alpha microprocessor, two entire layers of metal are dedicated for shielding [4]. A common method of shielding is placing ground or power lines at the sides of a victim signal line to reduce noise and delay uncertainty [5]. Although shielding is commonly used [6], [7], a peak noise model for shielded interconnects has not yet been developed. The crosstalk between two coupled interconnects is

This research is supported in part by the Semiconductor Research Corporation under Contract No. 99-TJ-687 and No. 2003-TJ-1068, the DARPA/ITO under AFRL Contract F29601-00-K-0182, grants from the New York State Office of Science, Technology \& Academic Research to the Center for Advanced Technology - Electronic Imaging Systems and to the Microelectronics Design Center, and by grants from Xerox Corporation, IBM Corporation, Intel Corporation, Lucent Technologies Corporation, Eastman Kodak Company, and Photon Vision Systems, Inc. often neglected when a shield is inserted, significantly underestimating the coupling noise. The crosstalk noise between two shielded interconnects can produce a peak noise of $15 \%$ of $V_{d d}$ in a $0.18 \mu \mathrm{m}$ CMOS technology. An accurate estimate of the peak noise for shielded interconnects is therefore necessary. This paper deals with crosstalk noise based on a proposed pseudo- $2 \pi R C$ model. The effects of the shield width, length, separation between the shield and the signal, and the number of connections tieing the shield to ground on the crosstalk noise are investigated.

In Section II, an analytic model of the peak noise is developed for shielded interconnects based on a pseudo$2 \pi R C$ model. In Section III, the analytic peak noise model is compared to SPICE, producing an average error of $4.4 \%$. In Section IV, the minimum number of ground connections for a target shield line with noise constraints is determined based on the peak noise model, following by a comparison of the effects on reducing coupling noise by either inserting a shield line or by increasing the physical separation between two coupled interconnects. Some conclusions are offered in Section V.

\section{Analytic Model of Crosstalk}

An interconnect structure composed of two shielded signal lines is shown in Fig. 1. The victim signal line is shielded by a ground or power line from the aggressor signal line.

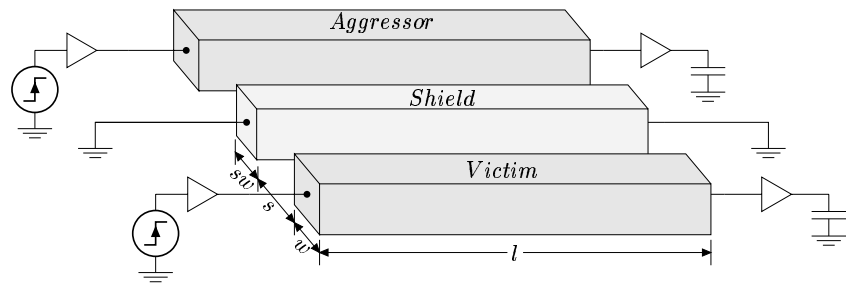

Fig. 1. Interconnect structure composed of two shielded signal lines

A proposed pseudo- $2 \pi R C$ model is used to model this interconnect structure, as shown in Fig. 2. The shield line is modeled as a $2 \pi R C$ line with the two ends tied to ground rather than an ideal ground.

The pseudo- $2 \pi R C$ model is different from a standard $2 \pi R C$ model by the manner in which the coupling capacitance at the receiver ends is modeled. In a standard $2 \pi R C$ model, the coupling capacitance at the receiver ends are shorted to ground, significantly underestimating the coupling 
noise. In the pseudo- $2 \pi R C$ model, these coupling capacitances between nodes $V_{j r 1}$ and $V_{j r 2}\left(V_{j r 2}\right.$ and $\left.V_{j r 3}\right)$ at the receiver ends are shifted to nodes $V_{j m 1}$ and $V_{j m 2}\left(V_{j m 2}\right.$ and $V_{j m 3}$ ), as shown in Fig. 2.

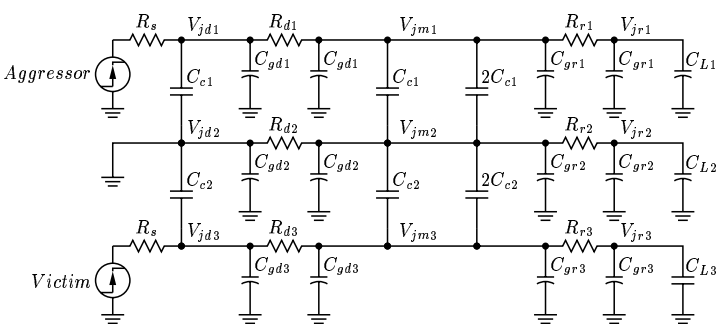

Fig. 2. Equivalent circuit model of pseudo- $2 \pi R C$ model

Consider crosstalk noise at the receiver end of a victim line where the aggressor $V_{a g g}$ switches from 0 to $V_{d d}$ and the victim is at either $G n d$ or $V_{d d}$. To determine the coupling noise $V_{j r 3}$ on the victim line, the transfer function $H_{1}(s)=\frac{V_{j m 2}(s)}{V_{a g g}(s)}$ of the circuit shown in Fig. 2 is obtained, followed by the transfer function $H_{2}(s)=\frac{V_{j r 3}(s)}{V_{j m 2}(s)}$ for the coupling noise at the receiver end of the victim line. Using a dominant-pole truncation approximation, both of the transfer functions, $H_{1}(s)$ and $H_{2}(s)$, can be modeled as a one pole system,

$$
\begin{aligned}
& H_{1}(s)=\frac{t_{x 1} s}{1+t_{y 1} s}, \\
& H_{2}(s)=\frac{t_{x 2} s}{1+t_{y 2} s} .
\end{aligned}
$$

Let

$$
\begin{aligned}
C_{x 1} & =3 C_{c 1}, \\
C_{x 2} & =3 C_{c 2}, \\
C_{1} & =C_{g d 1}+C_{c 1}, \\
C_{2} & =C_{g d 1}+C_{g r 1} \equiv 2 C_{g d 1}, \\
C_{3} & =C_{g r 1}+C_{L 1}, \\
C_{s} & =C_{g r s}+C_{g d s} \equiv 2 C_{g d s}, \\
C_{4} & =C_{g d 3}+C_{c 2}, \\
C_{5} & =C_{g d 3}+C_{g r 3} \equiv 2 C_{g d 3}, \\
C_{6} & =C_{g r 3}+C_{L 3} .
\end{aligned}
$$

$t_{x 1}, t_{y 1}, t_{x 2}$, and $t_{y 2}$ can be expressed as

$$
\begin{aligned}
t_{x 1}= & \frac{1}{2} C_{x 1} R_{d s} \\
t_{y 1}= & R_{s 1}\left(C_{1}+C_{2}+C_{3}+C_{x 1}\right)+R_{d 1}\left(C_{2}+C_{3}+C_{x 1}\right) \\
& +R_{r 1} C_{3}+R_{s 3}\left(C_{4}+C_{5}+C_{6}+C_{x 2}\right)+R_{r 3} C_{6} \\
& +R_{d 3}\left(C_{5}+C_{6}+C_{x 2}\right)+\frac{1}{2} R_{d s}\left(C_{s}+C_{x 1}+C_{x 2}\right) \\
t_{x 2}= & \left(R_{s 3}+R_{d 3}\right) C_{x 2} \\
t_{y 2}= & R_{s 3}\left(C_{4}+C_{5}+C_{6}+C_{x 2}\right)+R_{r 3} C_{6} \\
& +R_{d 3}\left(C_{5}+C_{6}+C_{x 2}\right)
\end{aligned}
$$

The physical meaning of $t_{x 1}, t_{y 1}, t_{x 2}$, and $t_{y 2}$ is

$t_{x 1} \quad R C$ delay of the shield line, the coupling capacitance $C_{x 1}$ times the effective resistance from node $V_{j m 2}$ to ground.

$t_{y 1} \quad$ the sum of the Elmore delays of all three nets.

$t_{x 2} \quad R C$ delay of the victim line, the coupling capacitance $C_{x 2}$ times the effective resistance from node $V_{j m 3}$ to ground. $t_{y 2} \quad$ Elmore delay of the victim line.

For an aggressor with a ramp input signal with a normalized power supply $V_{d d}$ and a transition time $t_{r}$,

$$
V_{\text {agg }}(t)=\left\{\begin{array}{lll}
t / t_{r} & \cdots & \text { for } 0 \leq t \leq t_{r} \\
1 & \cdots & \text { for } t>t_{r}
\end{array}\right.
$$

The Laplace transform is

$$
V_{a g g}(s)=\frac{1-e^{-s t_{r}}}{s^{2} t_{r}} .
$$

Combining (1), (2), and (17), the coupling noise in the victim line is

$$
V_{j r 3}(s)=H_{1}(s) H_{2}(s) V_{a g g}(s) \equiv \frac{t_{x 1} t_{x 2}\left(1-e^{-s t_{r}}\right)}{t_{r}\left(1+s t_{y 1}\right)\left(1+s t_{y 2}\right)} .
$$

Computing the inverse Laplace transform of (18), the coupling noise $V_{j r 3}(t)$ in the time domain is

$$
V_{j r 3}(t)=\left\{\begin{array}{l}
\frac{t_{x 1} t_{x 2}}{t_{r}\left(t_{y 1}-t_{y 2}\right)}\left(e^{-t / t_{y 1}}-e^{-t / t_{y 1}}\right), \quad t \leq t_{r} \\
\frac{t_{x 1} t_{x 2}}{t_{r}\left(t_{y 1}-t_{y 2}\right)}\left(a e^{-t / t_{y 1}}-b e^{-t / t_{y 2}}\right), \quad t>t_{r}
\end{array}\right.
$$

where $a=\left(1-e^{t_{r} / t_{y 1}}\right)$ and $b=\left(1-e^{t_{r} / t_{y 2}}\right)$. To determine the peak noise, (19) and (20) is differentiated with respect to $t$ and set equal to zero. The times at which the peak noise occur are

$$
\begin{aligned}
t_{\text {peak } 1} & =\frac{t_{x 1} t_{x 2}}{\left(t_{y 1}-t_{y 2}\right)} \ln \left(t_{y 1} / t_{y 2}\right), \quad 0<t_{\text {peak } 1}<t_{r} \\
t_{\text {peak } 2} & =\frac{t_{x 1} t_{x 2}}{\left(t_{y 1}-t_{y 2}\right)} \ln \left(b t_{y 1} / a t_{y 2}\right), \quad t_{\text {peak } 2}>t_{r} \\
t_{\text {peak } 3} & =t_{r}
\end{aligned}
$$

From (21), (22), and (23), the peak noise voltage can be computed by substituting $t_{\text {peak }}$ into (19) or (20). The peak noise for a shielded interconnect, therefore, is

$$
V_{j r 3}\left(t_{p e a k}\right)=\max \left\{V_{j r 3}\left(t_{p e a k 1}\right), V_{j r 3}\left(t_{p e a k 2}\right), V_{j r 3}\left(t_{r}\right)\right\} .
$$

\section{MODEL FIDELITY AND ACCURACY}

Some simulated results are presented in this section to verify the peak noise model shown in (24). For two coupled interconnects with a shield between the lines, as shown in Fig. 1, the ground capacitances, coupling capacitances, and resistances of each net are extracted using the OEA extraction tool NETAN [8]. A $0.18 \mu \mathrm{m}$ CMOS technology with $V_{d d}=1.2$ volts is assumed with this interconnect structure. The transition time of the input clock signal is $50 \mathrm{ps}$, and the $W / L$ ratio of the drivers and receivers are 100. The peak noise obtained from the analytical model is compared to SPICE in terms of the coupling length $l$, the shield line width $s w$, the signal line width $w$, and the physical separation $s$ between the signal line and the shield line, as shown in Figs. 3, 4, 5, and 6, respectively.

From these figures, the coupling noise for shielded interconnect increases with longer lines, and decreases with increasing shield width and physical separation between the signal line and the shield line. When the signal width increases, the resistance of the aggressor is reduced and the coupling noise increases. The ground capacitance of the victim line also increases, however, causing the coupling noise to decrease. 


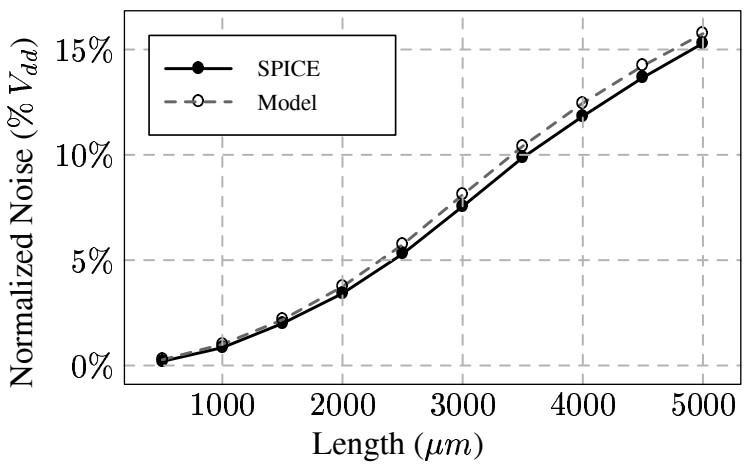

Fig. 3. Comparison of coupling noise using SPICE with the analytic model for three line lengths varying from $1500 \mu \mathrm{m}$ to $5000 \mu \mathrm{m}$. The maximum error of the peak noise model as compared to SPICE is $9.5 \%$. ( $w=0.8 \mu \mathrm{m}$, $s w=0.2 \mu m$, and $s=0.2 \mu m$ )

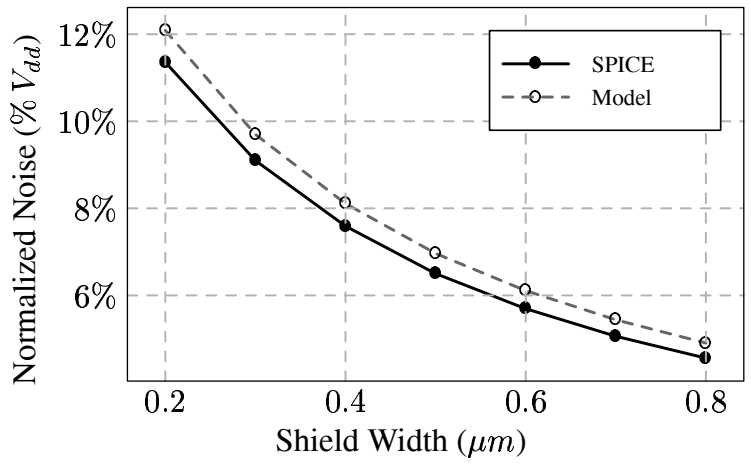

Fig. 4. Comparison of coupling noise using SPICE with the analytic model for the shield line width varying from $0.2 \mu \mathrm{m}$ to $0.8 \mu \mathrm{m}$. The maximum error of the peak noise model as compared to SPICE is 7.6\%. $(w=0.8 \mu \mathrm{m}$, $s=0.4 \mu \mathrm{m}$, and $l=5000 \mu \mathrm{m}$ )

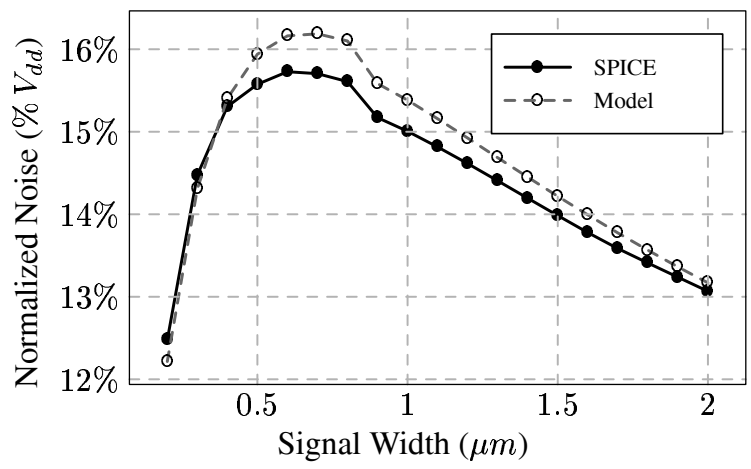

Fig. 5. Comparison of coupling noise using SPICE with the analytic model for the signal line width varying from $0.2 \mu \mathrm{m}$ to $2 \mu \mathrm{m}$. The maximum error of the peak noise model as compared to SPICE is $3.15 \%$. ( $s=0.2 \mu \mathrm{m}$, $s w=0.2 \mu m$, and $l=5000 \mu m$ )

Note that the peak noise model tracks the simulation results quite well. The model exhibits an average error of $4.4 \%$ as compared to SPICE.

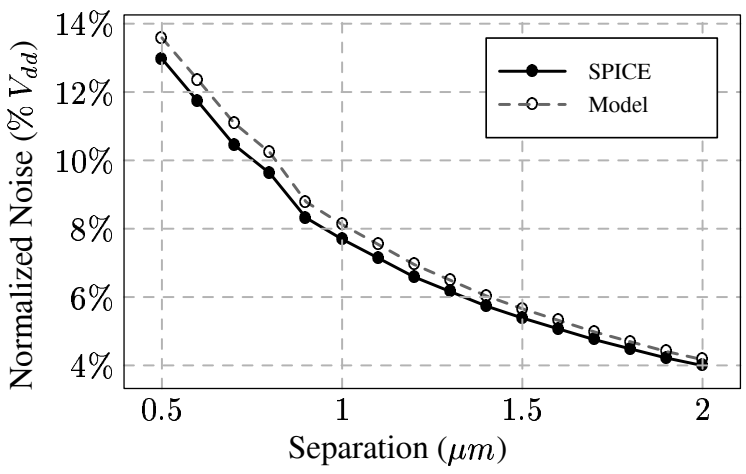

Fig. 6. Comparison of coupling noise using SPICE with the analytic model for the separation between the signal line and the shield line varying from $0.5 \mu \mathrm{m}$ to $2 \mu \mathrm{m}$. The maximum error of the peak noise model as compared to SPICE is $6.3 \%$. $(w=0.8 \mu m, s w=0.2 \mu m$, and $l=5000 \mu m)$

\section{DESIGN IMPLICATIONS}

In this section, the peak noise model is used to determine the minimum number of connections required to tie the shield line to the power/ground grid in order to satisfy a target noise constraint. A comparison of the effect on reducing noise by either inserting a shield line or by increasing the physical separation is also described in this section.

\section{A. Minimum Number of Ground Connections}

A shield line is not an ideal ground because of the parasitic resistance of the line which causes noise to couple to the victim signal line. As shown in Fig. 8, the greater the number of connections tieing the shield line to the power/ground grid, the smaller the coupling noise on the victim signal. In order to satisfy the coupling noise constraints, a minimum or greater number of ground connections is required.

The peak noise model described in (24) is for a shielded interconnect structure with a shield line grounded only at two ends. To determine the peak noise of a shielded interconnect structure with multiple ground connections in the shield line, the interconnect structure is divided into smaller parts in which the shield line is grounded only at two ends, as shown in Fig. 7. The peak noise of the shielded interconnect structure with a multiple grounded shield line is almost the same as the peak noise of the divided interconnect structure, as shown in Fig. 8 , and therefore can be determined from (24).

For a target noise constraint $V_{\max }$, the minimum number of ground connections for a shielded interconnect structure with length $l$ is

$$
N_{\text {connections }}=\frac{l}{l_{e q}},
$$

where $l_{e q}$ is the maximum length which satisfies

$$
V_{\text {noise }}\left(l_{e q}\right)<V_{\max } \text {. }
$$

\section{B. Increasing the separation vs. inserting a shield}

The techniques of increasing the physical separation as compared to inserting a shield to reduce crosstalk noise is 


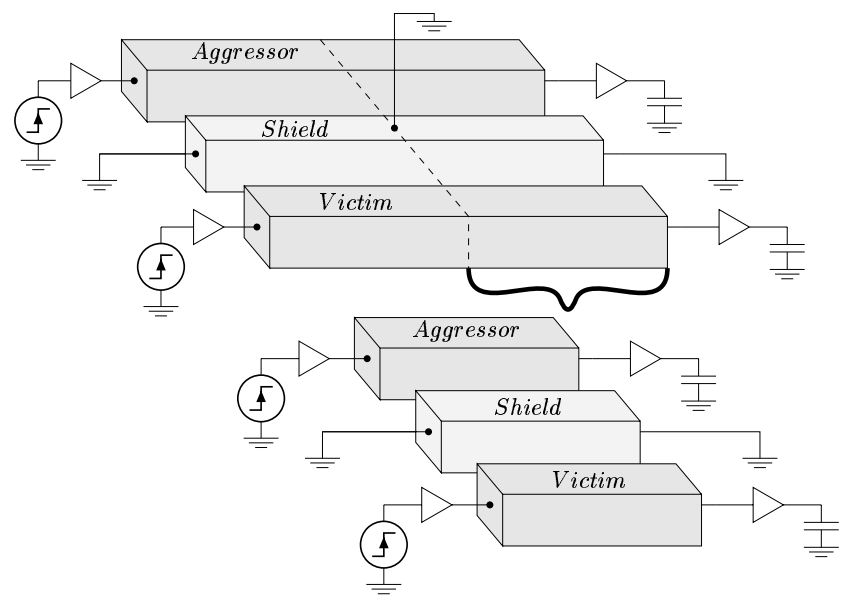

Fig. 7. The ground connection in a shield line is used to divide an interconnect structure with a multiple grounded shield line into a smaller interconnect structure with a shield line grounded at two ends. The coupling noise of these two interconnect structures is almost identical.

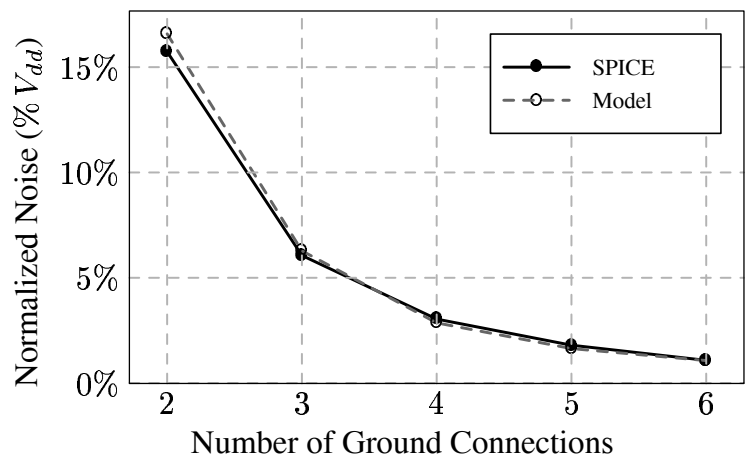

Fig. 8. The peak noise decreases with increasing number of ground connections. The peak noise of a shielded interconnect structure with a multiple grounded shield line is almost the same as the peak noise of the divided interconnect structure with the shield line grounded at only the two ends. The maximum error of the model as compared to SPICE is $9.3 \%$ $(l=5000 \mu m, w=0.6 \mu m, s=0.2 \mu m, s w=0.2 \mu m)$

discussed in this subsection. For two coupled interconnects with a separation $s$ and a shield width $s w$, as shown in Fig. 1 , the separation between signal lines in coupled interconnect without a shield is $s^{\prime}=2 * s+s w$.

The noise produced by shielded and unshielded interconnects can be obtained from the peak noise model (24) and the peak noise models described in [2] and [3], respectively. The reduction in noise achieved by increasing the physical separation and by inserting a shield are compared in Fig. 9. As shown in Fig. 9, the reduction in noise achieved by inserting a shield is much greater than by increasing the physical separation. If the space between the signal lines is sufficient for a minimum width shield line, inserting a shield is more efficient in reducing noise than increasing the physical separation.

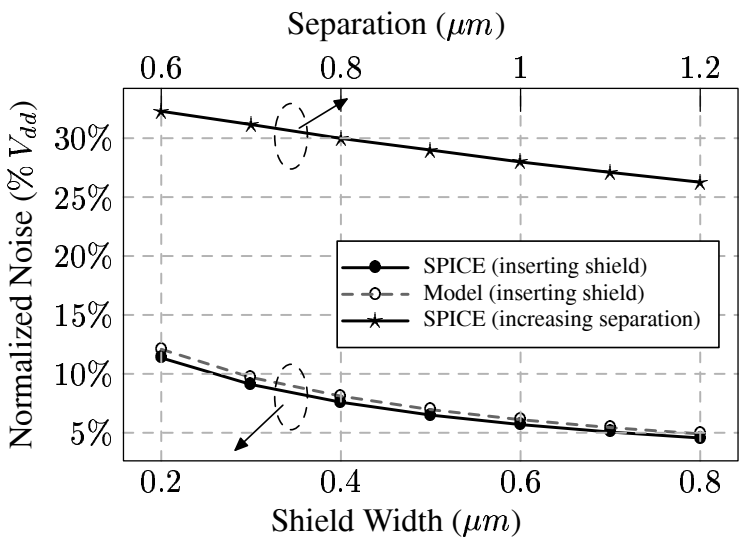

Fig. 9. Comparison of noise reduction techniques by either inserting a shield (bottom axis in the graph) or increasing the physical separation (top axis in the graph). $(l=5000 \mu m, w=0.8 \mu m$, and $s=0.4 \mu m)$

\section{Conclusions}

An analytic model of the peak noise for coupled $R C$ interconnects with a shield between the lines is proposed in this paper. With this peak noise model, the effects of the shield length, shield width, width of the signal line, and separation between the shield line and the signal line on the crosstalk noise are investigated. The peak noise model exhibits an average error of $4.4 \%$ as compared to SPICE. For a target shield line with noise constraints, a minimum number of connections required to tie the shield to ground can be obtained from this noise model. Simulation results show that inserting a shield is more effective in reducing coupling noise than increasing the physical separation.

\section{REFERENCES}

[1] A. Vittal, L. H. Chen, S. M. Marek, K.-P. Wang, and X. Yang, "Crosstalk in VLSI Interconnections," IEEE Transactions on Computer-Aided Desgin of Integrated Circuits and Systems, Vol. 18, No. 12, pp. 1817 1824, December 1999.

[2] K. T. Tang and E. G. Friedman, "Peak Crosstalk Noise Estimation in CMOS VLSI Circuits," Proceedings of the IEEE International Conference on Electronics, Circuits and Systems, pp. 1539-1542, September 1999.

[3] J. Cong, D. Z. Pan, and P. V. Srinivas, "Improved Crosstalk Modeling for Noise Constrained Interconnect Optimization," Proceedings of the IEEE Asia South Pacific Design Automation Conference, pp. 373-378, January 2001.

[4] D. W. Bailey and B. J. Benschneider, "Clocking Design and Analysis for a 600-Mhz Alpha Microprocessor," IEEE Journal of Solid-State Circuits, Vol. 33, No. 11, pp. 1627-1633, November 1998.

[5] Y. Im and K. Roy, "CASh: a Novel "Clock As Shield" Design Methodology for Noise Immune Precharge-evaluate Logic," Proceedings of the IEEE International Conference on Computer-Aided Design, pp. 337-341, November 2001.

[6] G. Yee, R. Christopherson, T. Thorp, B. Wong, and C. Schen, "An Automated Shielding Algorithm and Tool for Dynamic Circuits," Proceedings of the IEEE International Symposium on Quality Electronic Design, pp. 369-374, March 2000.

[7] B. M. Averill and K. G. Barkley, "Chip Integration Methodology for the IBM S/390 G5 and G6 Custom Microprocessors," IBM Journal of Research and Development, Vol. 43, No. 5, pp. 681-706, September 1999.

[8] OEA, NETAN Multi-Net Three Dimensional Field Solver Extraction Tool User Reference Manual, OEA International Inc., 2001. 